\title{
Wandering Fibroid: An Unusual Complication Arising from an Unusual Location
}

\author{
Nilu Malpani Dhoot ${ }^{1}$ Gobindo Pramanick ${ }^{1}$ Manash Saha ${ }^{1} \quad J$ Kishor Gupta ${ }^{2}$ Milan Chetry \\ Chandrashish Chakraborty ${ }^{3}$ Nipanjan Ghosh ${ }^{2}$ Arpita Sutradhar ${ }^{4}$
}

1Department of Radiodiagnosis and Intervention, Apollo Gleneagles Hospital, Kolkata, West Bengal, India

2Department of Gynecology, Apollo Gleneagles Hospital, Kolkata, West Bengal, India

${ }^{3}$ Department of Medicine, Apollo Gleneagles Hospital, Kolkata, West Bengal, India

${ }^{4}$ Department of Pathology, Apollo Gleneagles Hospital, Kolkata, West Bengal, India

J Gastrointestinal Abdominal Radiol ISGAR 2018;1:65-68

Address for correspondence Nilu Malpani Dhoot, DNB, DMRD, Department of Radiodiagnosis and Intervention, Apollo Gleneagles Hospital, Kolkata 700054, West Bengal, India (e-mail: drniludhoot@gmail.com).
Abstract
Keywords
- parasitic fibroid
- inferior epigastric artery embolization
- wandering fibroids

Wandering fibroids are exceedingly uncommon extrauterine neoplasms. They occur in unusual locations that can confound imaging and diagnosis. We present a case of a woman, with a lump in the abdomen, whose imaging revealed uterine fibroids and an apparently unrelated solid mass adherent to the anterior abdominal wall. Guided biopsy resulted in the unusual complication of an inferior epigastric artery branch injury for which embolization was done. Post embolization, the size of the mass reduced. latrogenic inferior epigastric artery injury is rare and can result in a large rectus sheath hematoma. Active bleeding is life threatening and warrants immediate intervention.

\section{Introduction}

Parasitic leiomyoma or "wandering fibroid" (WF) is an extremely infrequent extrauterine benign neoplasm with unusual locations and presentations that can confound imaging and require a histopathological diagnosis. ${ }^{1}$ We report a case of a percutaneous biopsy proven WF. There was injury to a branch of the inferior epigastric artery (IEA) during biopsy, resulting in a large rectus sheath hematoma. Spontaneous rupture, trauma, and iatrogenic injury can all cause these rare IAE bleeds, with iatrogenic injury occurring most often. ${ }^{2}$ Urgent therapeutic embolization or surgery may be required as a life-saving measure. Our patient underwent an IAE embolization. On follow-up imaging, there was reduction in the size of both the hematoma and the parasitic fibroid (PF), obviating the need for further treatment in this asymptomatic perimenopausal woman.

\section{Case}

A 48-year-old, parous, perimenopausal woman presented with lower abdominal heaviness and swelling for 6 months that worsened on exercise and was associated with mild occasional pain. There were no symptoms localizing to a gynecological pathology. Physical examination revealed a well-defined, fixed, firm 9-cm mass in the left paraumbilical region under normal overlying skin. Ultrasonography (USG) revealed an enlarged uterus with multiple intramural fibroids. In addition, there was a well-defined heterogeneously isoechoic $9 \times 8 \times 7 \mathrm{~cm}$ solid nonspecific mass adherent to the anterior abdominal wall, with no significant vascularity on Doppler. Although an MRI (magnetic resonance imaging) was recommended to further characterize the lesion, contrast-enhanced computed tomography (CECT) was done because of attendant claustrophobia.

Contrast-enhanced multidetector CT (Brilliance Big Bore, Philips, Holland, the Netherlands.) abdomen was taken. Precontrast and postcontrast (arterial: 20 seconds, venous: 60 seconds, delayed: 5 minutes) $1-\mathrm{mm}$ thin sections were acquired from the domes of the diaphragm to just below the pubic symphysis following administration of low osmolar nonionic contrast (Omnipaque 300, Boston, United States) at the dose of $1.5 \mathrm{~mL} / \mathrm{kg}$ and rate of $3 \mathrm{~mL} / \mathrm{sec}$.

On CT, the mass was circumscribed, oval, solid, heterogeneously enhancing (HU [Hounsfield unit]
$10.1055 / \mathrm{s}-0038-1675324$
(C)2018 by Indian Society of Gastrointestinal and Abdominal Radiology
License terms

$($ (1) $\Theta \circledast$ 
maximum intensity projection 87 ), and attached to the inner surface of the left rectus abdominis muscle of the anterior abdominal wall at the left paraumbilical region. In addition, the uterus was enlarged and revealed multiple homogeneously enhancing solid masses suggestive of fibroids. Although similar in appearance to uterine fibroids inferiorly, the mass was clearly separated from the uterus by intraperitoneal fat and intervening small bowel (-Fig. 1). A differential diagnosis of WF, gastrointestinal stromal tumor and desmoid was made. Since the management of these entities is different, a definite histopathological diagnosis was sought. USG-guided core biopsy was performed using an $18 \mathrm{G}$ coaxial gun (Bard Mission Tempe, AZ). Three tissue cores were obtained and the tract sealed with gel foam. The procedure was uneventful; however, within 30 minutes, the patient complained of severe pain and a rapidly increasing swelling at the biopsy site. Urgent CT angiography revealed active contrast extravasation from a tiny branch of the left inferior epigastric artery (-Fig. 2) within a $15 \mathrm{~cm}$ left rectus sheath hematoma (-Fig. 2A). Due to significant lowering of hematocrit, an urgent embolization of the left IEA was done using two coils $(2 \times 2 \mathrm{~cm}$, Hilal, Cook Medical System, Bloomington, IN) and gel foam (-Fig. 3); stasis and no active contrast leak were taken as endpoints during embolization. Post embolization, the patient was transfused with two units of packed red blood cells. There was no further increase in size of the hematoma and the patient was discharged after a few days in a hemodynamically stable condition and kept on regular follow-up.
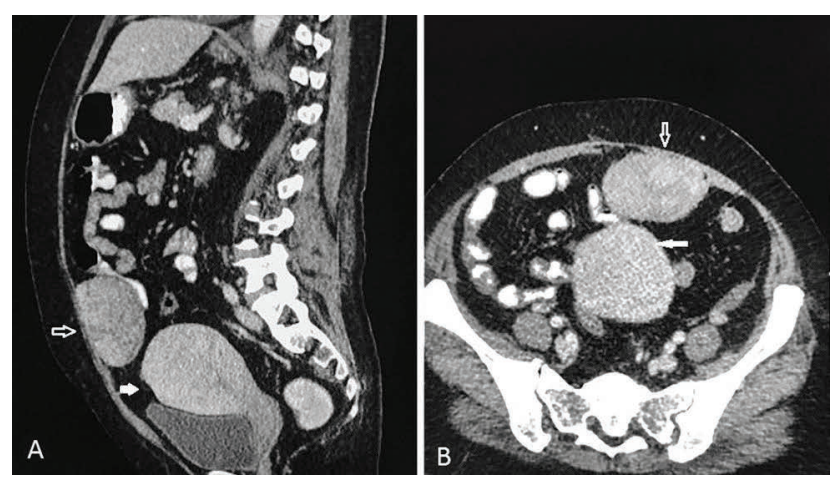

Fig. 1 (A) Oblique sagittal CECT and (B) axial CECT showing parasitic fibroid (arrow) and uterine fibroids (solid arrow). CECT, contrast enhanced computed tomography.
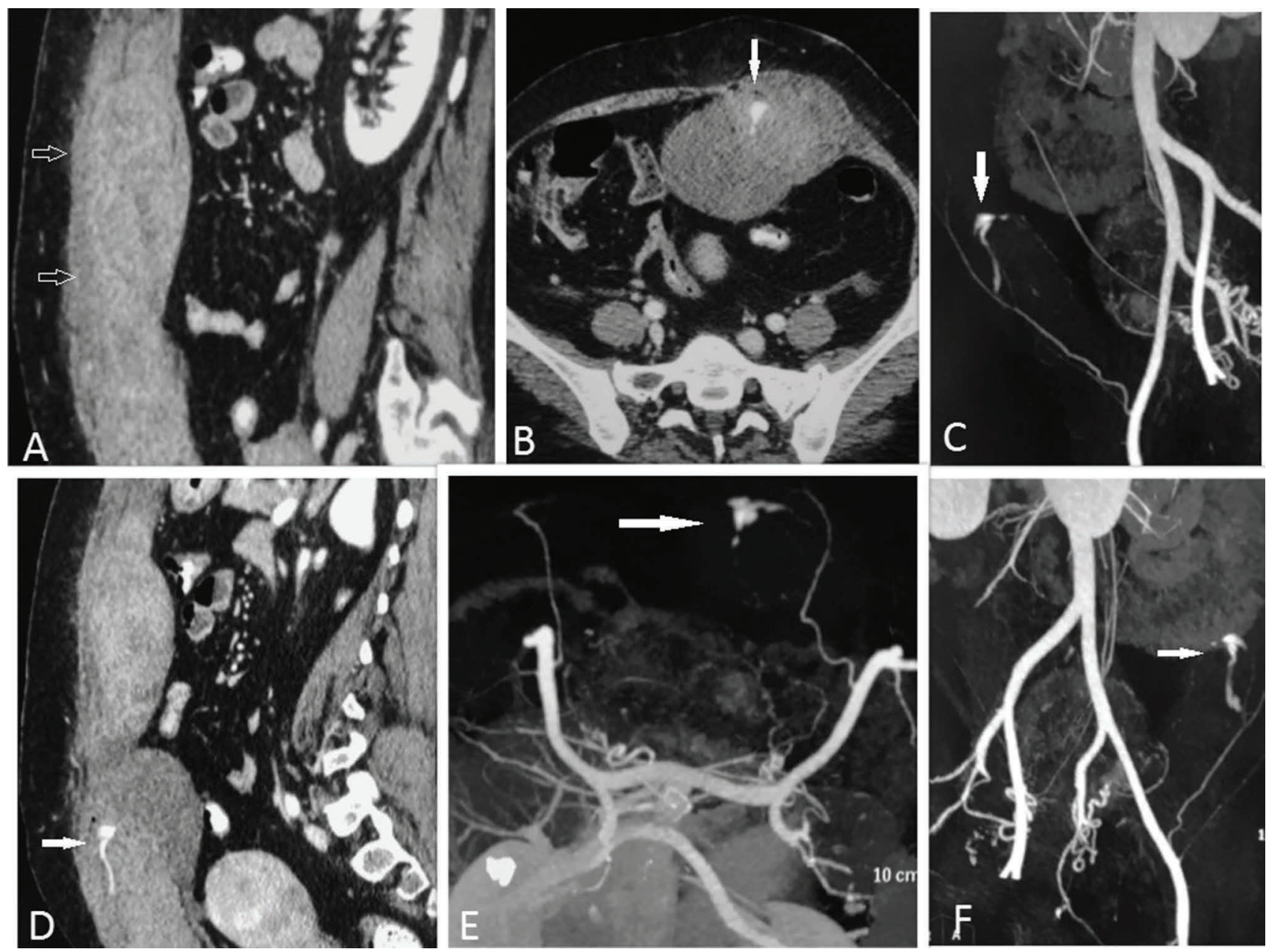

Fig. 2 (A) Sagittal CECT, (B) axial CECT, (D) oblique sagittal CECT, (C, E, and F) CT angiography MIP showing rectus sheath hematoma (arrow) and active contrast extravasation from a branch of the inferior epigastric artery (solid arrow). CECT, contrast enhanced computed tomography; MIP, maximum intensity projection. 
Histopathology revealed benign spindle cells, positive for smooth muscle actin, and desmin on immunohistochemistry, consistent with a benign leiomyoma ( - Fig. 4). Follow-up USG at 1 month showed partial resolution of the hematoma, as expected (-Fig. 5). The leiomyoma also demonstrated change with marginal reduction in size and the appearance of a few cystic spaces. Since the patient was asymptomatic and perimenopausal, no further treatment was offered for the PF or uterine fibroids and regular follow-up was advised.

\section{Discussion}

Parasitic or WFs are leiomyomas with no uterine connection or myometrial participation. ${ }^{3}$ They can be primary where a subserosal fibroid spontaneously detaches itself and acquires blood supply from adjacent organs or peritoneum. ${ }^{4,5}$ They are commonly iatrogenic due to seedling during

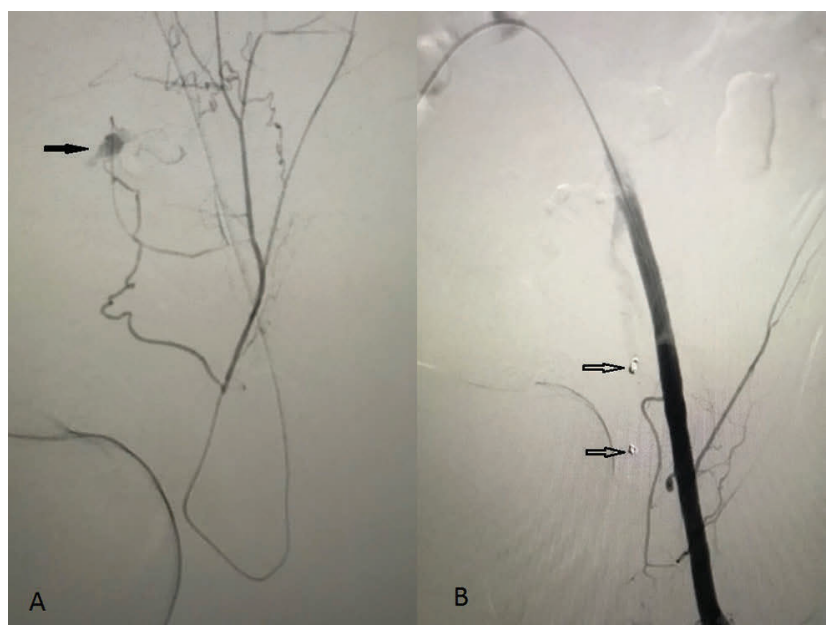

Fig. 3 (A) DSA (digital subtraction angiography) selective cannulation of inferior epigastric artery showing active contrast extravasation (arrow) and coils, (B) DSA post coil embolization showing no evidence of contrast extravasation.

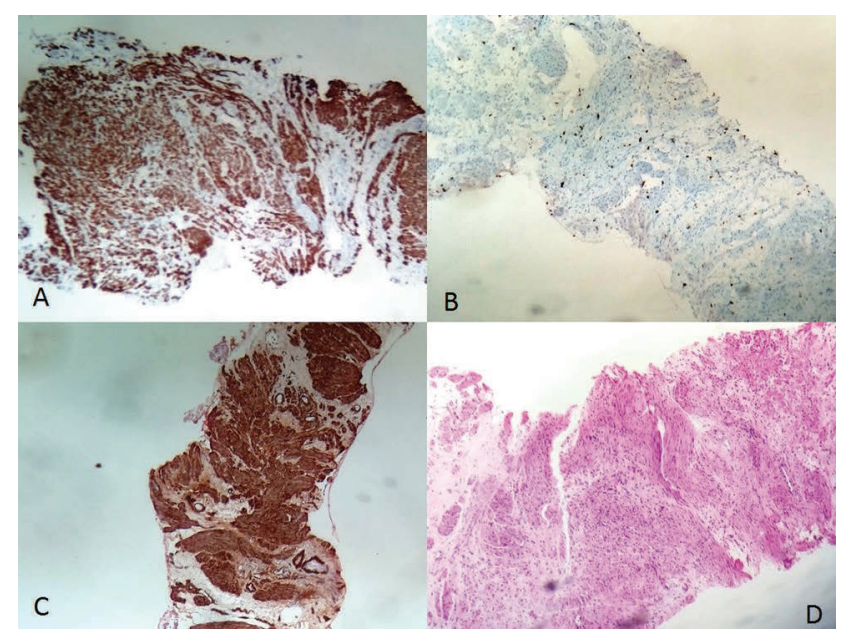

Fig. 4 (A) Immunohistochemistry diffusely positive for desmin, (B) immunohistochemistry for Ki-67 shows $2 \%$ positivity, (C) immunohistochemistry diffusely positive for smooth muscle actin, (D) Hematoxylin and eosin stain shows fascicles of round to oval benign spindle cells having elongated cytoplasm. myomectomy or hysterectomy. ${ }^{6}$ There are not many reported cases of parasitic leiomyoma. Most of the reported WFs are secondary iatrogenic. Lu et al presented 6 cases of postsurgical PFs. ${ }^{7}$ Gaspare et al concluded morcellation during hysterectomy as a risk factor in developing parasitic leiomyomas in a retrospective study. ${ }^{8}$ Our patient did not report prior abdominal procedures and is therefore a primary wandering leiomyoma. The incidence of primary PFs is exceedingly rare but a few cases have been reported.9,10 Concomitant occurrence of uterine and parasitic leiomyomas has also been reported as in our case. ${ }^{11}$ The unusual location, uncertain origin, and extrauterine blood supply of these PFs creates a diagnostic challenge on imaging. As in our case, a definitive histopathological diagnosis was needed to plan effective management. However, in our case, the process of tissue sampling resulted in a rare but potentially fatal complication of an IEA branch bleed. A retrospective review of IEA injuries presenting to interventional radiology departments of three university-affiliated hospitals in California, revealed only 20 cases over a 12 -year period, with a 30-day mortality rate of $30 \%{ }^{12}$ Sobkin et al reviewed 19 cases of intervention-related IAE injury and reported the following spectru paracentesis $40 \%$, surgical injury $5 \%$, percutaneous drain or biopsy injury $15 \%$, blunt trauma $10 \%$, subcutaneous injection 5\%, stabbing 5\%, and unknown and spontaneous $10 \%{ }^{2}$ The IEA originates from the external iliac artery; variation in its origin is not uncommon. ${ }^{13}$ After origin it ascends loosely between the rectus abdominis muscle and the posterior rectus sheath. The combination of the loose attachment of IEA with the stabilization of its perforating branches fixed to the muscle makes the artery prone to shearing stresses at branching sites even during strong muscular contraction or simple paracentesis. ${ }^{14}$ Conservative approaches, such as compression of the bleed are usually not effective because the abdomen lacks bony support and the bleeder is compressed against soft and mobile abdominal organs. Surgery or embolization is urgently required in cases of active bleeding because of the risk of rapid onset hypovolemic shock. We embolized the left IEA and achieved

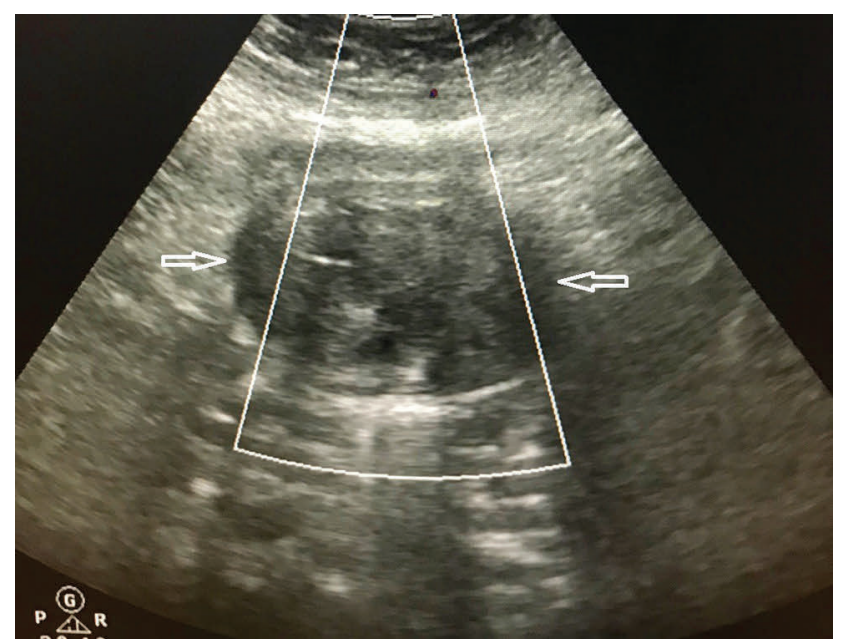

Fig. 5 Follow-up USG at 1 month showing no signal on Doppler; arrows point to wandering fibroid. USG, ultrasonography. 
hemostasis. Serendipitously, this intervention not only controlled the bleeding but also partially treated the disease.

\section{Conclusion}

Parasitic leiomyomas or WFs are rare and primary spontaneous PFs are rarer still. IEA injury is a life-threatening complication that should be kept in mind even while performing small needle biopsies particularly in the paraumbilical region. When there is active contrast extravasation on CECT or evidence of hemodynamic instability and a rapidly growing hematoma, urgent embolization should be immediately performed.

\section{Conflict of Interest}

None.

\section{References}

1 Salih AM, Kakamad FH, A H D, J Habibullah I, M Rauf G, Najar KA. Parasitic leiomyoma: A case report with literature review. Int J Surg Case Rep 2017;41:33-35

2 Sobkin PR, Bloom AI, Wilson MW, et al. Massive abdominal wall hemorrhage from injury to the inferior epigastric artery: a retrospective review. J Vasc Interv Radiol 2008;19(3):327-332

3 Berek JS. Uterine fibroids. In: Berek JS, ed. Berek \& Novak's Gynecology. 15th ed. Philadelphia, PA: Lipincott Williams \& Wilkins; 2013:444-500
4 Robbins SL, Cotran RS, Kumar V. Pathologic Basis of Disease. 3rd ed. Philadelphia, PA: W.B. Saunders; 1984:1109

5 Kelly HA, Cullen TS. Myomata of the Uterus. Philadelphia, PA: WB Saunders; 1909

6 Kho KA, Nezhat C. Parasitic myomas. Obstet Gynecol 2009;114(3):611-615

7 Lu B, Xu J, Pan Z. Iatrogenic parasitic leiomyoma and leiomyomatosis peritonealis disseminata following uterine morcellation. J Obstet Gynaecol Res 2016;42(8):990-999

8 Gaspare C, Roberta G, Gloria C, Edgardo S. Parasitic myomas after laparoscopic surgery: an emerging complication in the use of morcellator? Description of four cases. Fertil Steril 2011;96(2):90-96

9 Zaitoon MM. Retroperitoneal parasitic leiomyoma causing unilateral ureteral obstruction. J Urol 1986;135(1):130-131

10 Sarmalkar M, Nayak A, Singh N, Mehendale M, Dixit P. A rare case of primary parasitic leiomyoma mimicking as ovarian mass: a clinical dilemma. Int J Reprod Contracept Obstet Gynecol 2016;5(2):545-548

11 Nappi L, Bettocchi S, Carriero C, Ceci O, Vimercati A, Resta L. Large parasitic leiomyoma of the broad ligament. J Gynecol Surg 2004;20(3):97-102

12 Sobkin PR, Bloom AI, Wilson MW, et al. Massive abdominal wall hemorrhage from injury to the inferior epigastric artery: a retrospective review. J Vasc Interv Radiol 2008;19(3):327-332

13 Williams PL. Gray's Anatomy. 38th ed. Edinburgh: Churchill Livingstone; 1995:1563

14 Aggarwal P, Kangjam P, Singh MR, Akoijam U. Inferior epigastric artery injury during vaginal delivery. Int J Sci Res 2015;4(5):688 